\title{
FOUR-QUADRANT LOCAL ANAESTHESIA TECHNIQUE FOR VITREORETINAL SURGERY
}

\author{
J. D. STEVENS ${ }^{1}$, W. A. FRANKS ${ }^{2}$, G. ORR ${ }^{1}$, P. K. LEAVER ${ }^{1}$, R. J. COOLING ${ }^{1}$ \\ London
}

\begin{abstract}
SUMMARY
We report our experience of a recently described local anaesthetic technique which seeks to avoid risk of perforation of the globe, damage to the optic nerve, or injection into the subarachnoid space, whilst providing prolonged and reliable anaesthesia. A prospective series of 19 patients who underwent vitreoretinal surgery using this technique were compared with 19 patients who had retrobulbar anaesthesia for cataract extraction. The vitreoretinal group had excellent akinesia and very good anaesthesia, allowing prolonged retinal reattachment surgery lasting up to 3 hours. Patient evaluation of discomfort or pain experienced in the two groups was assessed using a visual analogue pain score chart. The pain scores for the two groups were not significantly different $(p=0.03)$ and 16 of 19 patients in each group $(84 \%)$ experienced only slight pain or less. Satisfaction with local anaesthesia, in both groups, was also assessed by asking patients which method of anaesthesia they would prefer if future surgery were to be performed. In the vitreoretinal group, 18 of 19 patients expressed a preference for local anaesthesia and in the cataract group 17 ot 19 also favoured local anaesthesia. The vitreoretinal patients' median pain score was 0 compared with 1 for the cataract patients. This study demonstrates that local anaesthesia provides pain relief for vitreoretinal surgery which is comparable to the experience of patients undergoing cataract surgery by retrobulbar anaesthesia. The technique described can provide successful local anaesthesia for vitreoretinal procedures. The success of this technique for pain relief and akinesia calls for a reappraisal of the number of patients suitable for vitreoretinal surgery under local anaesthesia.
\end{abstract}

Retrobulbar and peribulbar local anaesthesia have not found popularity in the UK for vitreoretinal surgery because of the difficulty in ensuring prolonged and reliable akinesia and anaesthesia. A recently described local anaesthetic technique ${ }^{1}$ seeks to avoid the risks of per-

From: 'Moorfields Eye Hospital, City Road, London EC1V 2PD, UK; ${ }^{2}$ St Thomas' Hospital, Lambeth Palace Road, London SE1, UK.

Correspondence to: Mr. J. D. Stevens, Moorfields Eye Hospital, City Road, London EC1V 2PD, UK. foration of the globe, damage to the optic nerve, and injection into the subarachnoid space whilst providing prolonged and dependable anaesthesia. Conventional retrobulbar or peribulbar local anaesthesia involves blind penetration with either a sharp or a relatively 'blunt' needle. The degree of ocular anaesthesia and akinesia achieved is unpredictable, due to variations in point of delivery and differences in diffusion of anaesthetic solution within the orbital tissues. There have been many reports of complications of sharp-needle local anaesthesia ${ }^{2-4}$ and any method of reducing these is to be welcomed.

The method of Mein and Woodcock ${ }^{1}$ involves a direct sub-Tenon's approach, using a blunt irrigation cannula, combined with a facial nerve block. The blunt cannula is guided around the globe posterior to the equator and local anaesthetic solution delivered. Direct infiltration without using a sharp instrument avoids the risk of ocular perforation and would be expected to reduce the incidence of orbital haemorrhage.

\section{METHOD}

Surgery under local anaesthesia was offered to patients with general medical disorders involving increased risk for general anaesthesia and to other patients on request. Four drops of topical amethocaine $1 \%$ were instilled in the operative eye and a local anaesthetic facial block given by the method of van Lint ${ }^{3}$. A lid speculum was placed and a subconjunctival injection of lignocaine $2 \%$ given, raising a small bleb, approximately a quarter of the way around the limbus. Three further injections were sufficient to raise a $360^{\circ}$ bleb. A $360^{\circ}$ limbal peritomy was then made, before separating the planes of Tenon's fascia to enter Tenon's space with blunt Westcott scissors. Tenon's space is a potential space between Tenon's fascia and the globe which has also been termed the episcleral space. A blunt 19 gauge visco-cannula was then guided in Tenon's space, backward along the contour of the globe in each quadrant (Fig. 1).

Local anaesthetic solution $(0.5-1.0 \mathrm{ml})$ was administered at the 1.30, 4.30, 7.30 and $10.30 \mathrm{o}$ 'clock positions, 


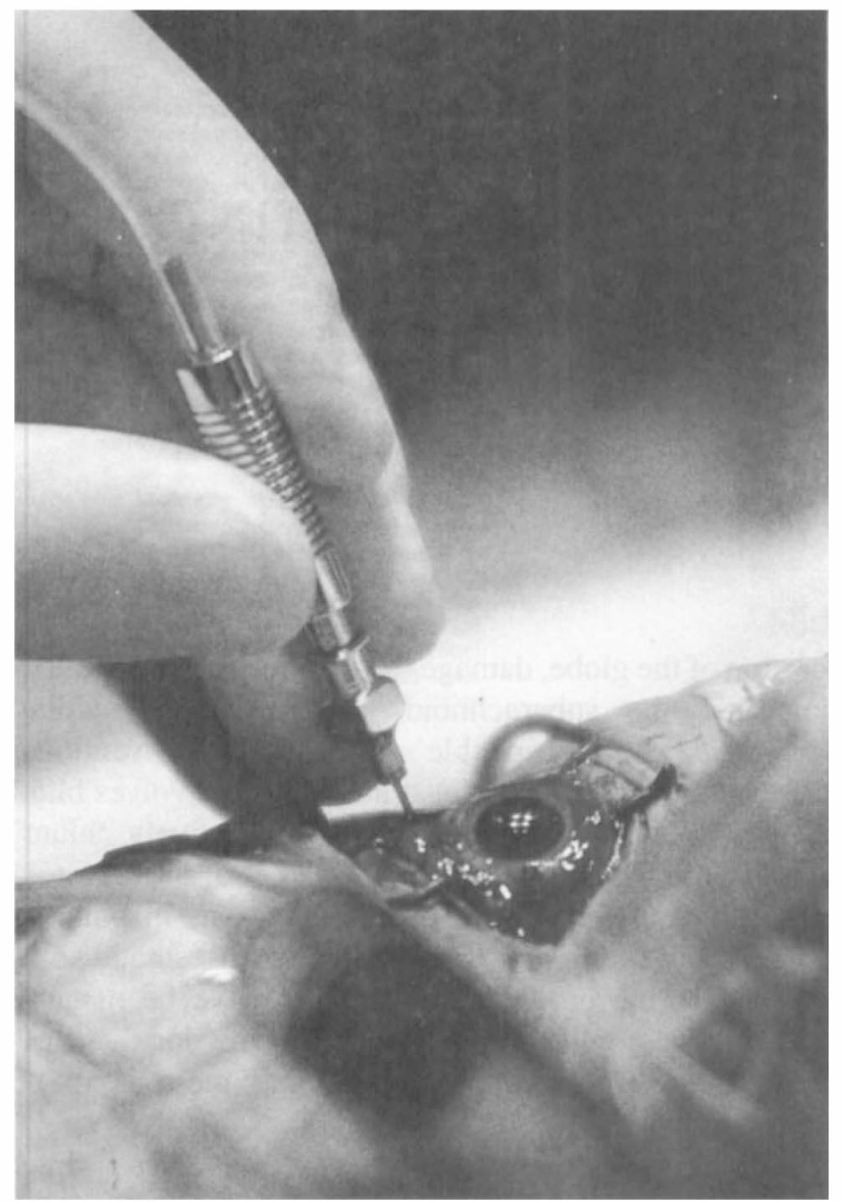

Fig. 1. A blunt visco-cannula attached to flexible tubing is used for the administration of four-quadrant local anaesthesia, the cannula being guided along the contour of the globe to deliver anaesthetic solution posterior to the equator of the eye.

between the rectus muscles. The globe slightly proptosed as the solution was given, providing a visible indicator that it was being delivered to the correct location. We used a 50:50 mixture of lignocaine $2 \%$ (plain) and bupivacaine $0.75 \%$ in a $10 \mathrm{ml}$ syringe from which a top-up of anaesthetic solution can easily be given per-operatively if required.

To assess the efficacy of the anaesthesia, patients were questioned regarding its delivery and any pain or discomfort felt during the subsequent operation. Post-operative

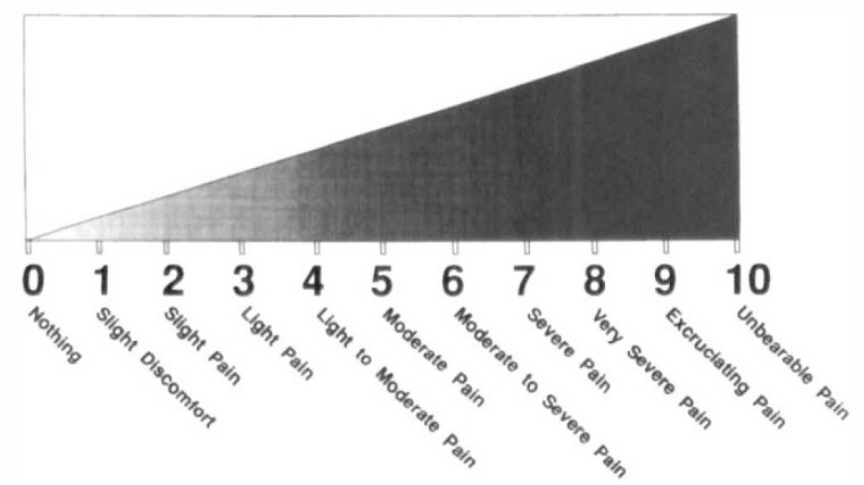

Fig. 2. Visual analogue $10 \mathrm{~cm}$ pain score chart with indicator pain descriptions. patients were shown a visual analogue pain score chart (Fig. 2) and asked to decide where on the scale they perceived any pain or discomfort felt during the operative procedure. If the patient had difficulty reading the chart a verbal rating from 0 to 10 was requested. This approach to pain assessment is commonly used. ${ }^{5-7}$

Information was recorded as to whether the patient had received a general anaesthetic previously for ocular or non-ocular surgery and whether, if a further ocular operation were required, the patient would choose local or general anaesthesia. The nature of the operative procedure was also recorded.

Nineteen patients who received a retrobulbar local anaesthetic for extracapsular cataract surgery were shown the same visual analogue graphical pain score chart and asked to grade any pain or discomfort experienced during the procedure in an identical manner.

\section{RESULTS}

Nineteen patients were entered into each group (Table I). The median pain score for the retinal patients was 0 (mean 0.8 ) and for the cataract group 1 (mean 1.1). There was no statistically significant difference between the means (Student's $t$-test and non-parametric Wilcoxon matched pairs signed ranks test, $p=0.03$ ), but the range of pain scores of the retinal group (0-8) was wider than that for the cataract group $(0-3)$. Fig. 3 charts the distribution of pain scores for the two groups, demonstrating that substantially more patients had a score of 0 in the retinal group $(n=12)$ than in the cataract group $(n=6)$. Sixteen patients in each group (84\%) had a pain score of less than 3 (slight pain or less). Pain scores were similar in 5 patients who had a 'conventional' retinal detachment repair and in 14 who underwent vitreoretinal procedures.

Of concern is the one patient in the retinal group who reported a score of 8 (very severe pain), with poor ocular akinesia. The surgeon was aware that the patient was experiencing pain and a top-up of local anaesthetic was given. No previous surgery had been performed and no abnormal tissue adhesion was encountered.

Retinal patients 12 hours post-operatively revealed marked deficits of ocular motility which had resolved on review the following day. Afferent pupillary defect was not assessed since many of the retinal patients had preoperative afferent defects, due to the nature of their pathology.

Table I. Summary of visual analogue pain score chart results

\begin{tabular}{lcc}
\hline & $\begin{array}{c}\text { Four-quadrant } \\
\text { retinal group }\end{array}$ & $\begin{array}{c}\text { Retrobulbar } \\
\text { cataract group }\end{array}$ \\
\hline Sample size & 19 & 19 \\
Mean & 0.84 & 1.08 \\
Median & 0 & 1 \\
Mode & 0 & 1 \\
Minimum & 0 & 0 \\
Maximum & 8 & 3 \\
Range & 8 & 3 \\
Comparison of the two sample groups & \\
Sample 1: retinal group $(n=19)$ & \\
Sample 2: cataract group $(n=19)$ & \\
2 $=-2.12(p=0.03)$ & \\
\hline
\end{tabular}




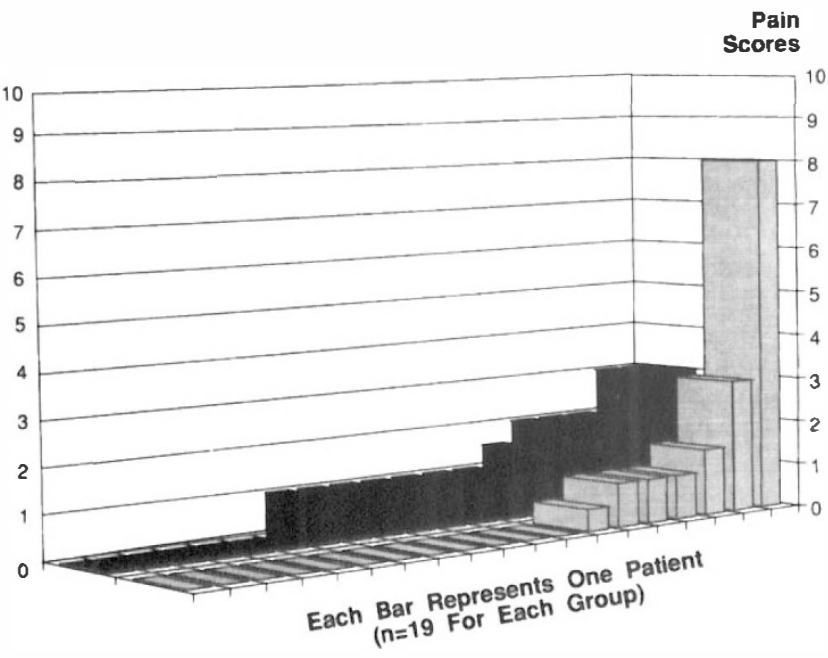

= Cataract Patient Pain Scores

= Retinal 4-Quadrant Anaesthesia Pain Scores

Fig. 3. Visual analogue pain scores comparing retinal with cataract patients.

In this study 18 of 19 retinal patients and 17 of 19 cataract patients would opt in future for local anaesthetic. Of the retinal patients, 10 had received general anaesthesia previously and were able to compare directly their experience of both forms of anaesthesia; all 10 preferred local anaesthesia. The patient with the pain score of 8 was the only retinal patient who would opt for general anaesthesia.

\section{DISCUSSION}

The use of local anaesthesia for vitreoretinal surgery has several advantages. It is often the case that vitreoretinal surgery is performed as an emergency, and it may be difficult to arrange in the face of a busy operating theatre timetable. Local anaesthesia has the advantage that patients do not need to be starved prior to surgery, unless conversion to general anaesthesia is a possibility and patients can go directly to the operating theatre. One patient in this series attended the casualty department in the afternoon, underwent surgery within 2 hours and was able to return home in the evening. Post-operative review was performed the following morning and the patient was delighted at not having to remain overnight in hospital.

Patients with sickle cell disease require special precautions when undergoing surgery with general anaesthesia. Local anaesthesia reduces the anaesthetic risk for this group of patients and allows prolonged vitreoretinal surgery to be performed. Those with other general medical disorders requiring urgent retinal surgery who are poor candidates for general anaesthesia would also benefit from a local procedure.

All patients had an ECG examination pre-operatively as well as per-operative cardiac monitoring. The oculo-cardiac reflex may manifest as reflex bradycardia, nausea and faintness when traction is applied to the extraocular muscles or pressure applied to the orbit. Retrobulbar anaesthesia is known to block motor aspects of the reflex ${ }^{8}$ and it may be partially or completely blocked by the fourquadrant anaesthesia. We advocate chest leads, however, attached to a cardiac monitor, for all patients. Some slowing of cardiac rate was encountered when traction sutures were being applied to extraocular muscles at the beginning of surgery (one patient converted from atrial fibrillation to sinus rhythm), but later in the procedures traction had little effect. Since traction sutures are applied at the beginning of vitreoretinal surgery, it may be that the anaesthetic combination has not fully blocked the oculocardiac reflex at this stage. Patients having conventional retinal detachment surgery, involving scleral buckling, generally undergo more muscle traction than those during vitrectomy without scleral buckling, though this did not appear to present any problem, nor were the pain scores different.

Patients who have had previous ocular surgery have tissue adhesions which may limit diffusion of the anaesthetic solution, while the presence of a scleral buckle may cause difficulty when guiding the blunt cannula along the contour of the globe, so that it may not be possible to insert the cannula directly into the sub-Tenon's space without some prior dissection of peri-ocular scar tissue. Vitreoretinal surgery under local anaesthesia, however, also requires the patient to remain still for an extended period and extraocular factors such as arthritis, backache and respiratory difficulties must be taken into consideration.

In this study 18 of 19 patients in the vitreoretinal group and 17 of 19 in the cataract group would opt for local anaesthesia for future surgery. Patients may, however, have a tendency to favour the form of anaesthesia with which they are familiar-if the outcome has been successful.

Effective anaesthesia was found to last for up to 12 hours, providing relief from immediate post-operative pain. Examination on the day after surgery revealed that some patients had deficits of ocular motility, but these resolved on later review. We used $0.75 \%$ bupivacaine with $2 \%$ lignocaine, the bupivacaine providing the long-acting component of the mixture. Their initial concentration is reduced when the solution is mixed, but appeared to be sufficient in all but one instance. The long-acting mixture has advantages post-operatively in reducing the pain or nausea experienced by many vitreoretinal patients. ${ }^{9,10}$

We have found that the four-quadrant local anaesthesia technique achieves pain relief for vitreoretinal surgery which is comparable to the experience of patients undergoing cataract surgery by retrobulbar anaesthesia. The technique can provide effective local anaesthesia for vitreoretinal procedures. The successful relief of pain and level of akinesia achieved, with the option of topping up the local, allows conventional re-attachment and vitreoretinal surgery to be performed. The introduction of this technique calls for a reappraisal of the number of patients suitable for retinal surgery under local anaesthesia.

Key words: Human, Local anaesthesia, Retinal detachment, Surgery, Sub-Tenon's anaesthesia, Vitreoretinal.

\section{REFERENCES}

1. Mein CE and Woodcock MG: Local anaesthesia for vitreoretinal surgery. Retina 1990, 10: 47-9. 
2. Ramsay RC and Knobloch WH: Ocular perforation following retrobulbar anaesthesia for retinal detachment surgery. Am J Ophthalmol 1978, 86: 61-4.

3. Feibel RM: Current concepts in retrobulbar anaesthesia. Surv Ophthalmol 1985, 30: 102-10.

4. Brookshire GE, Gleitsman KY, Shenk EC: Life-threatening complication of retrobulbar block. Ophthalmology 1986, 93: $1476-8$.

5. Ohnhaus EE, Adler R: Methodological problems in the measurement of pain: a comparison between the verbal rating scale and visual analogue scale. Pain 1975, 1: 375-84.

6. Revill SI, Robinson JO, Rosen M, Hogg MIJ: The reliability of a linear analogue for evaluating pain. Anaesthesia 1976, 31: 1191-8.

7. Scott J and Huskisson EC: Graphic representation of pain. Pain 1976, 2: 175-84.

8. Walsh FB and Hoyt WF: Sensory innervation of the eye and orbit. In: Clinical Neuro-ophthalmology, 3rd edn. Baltimore: Williams \& Wilkins, 1969.

9. Isemhagen RD, Michels RG, Glaser BM, de Bustros S, Enger C: Hospitalization requirements after vitreoretinal surgery. Arch Ophthalmol 1988, 106: 767-70.

10. Wilson D and Barr CC: Outpatient and abbreviated hospitalization for vitreoretinal surgery. Ophthalmic Surg 1990, 21: 119-22. 